\title{
Correlações genéticas de características de tamanho corporal e condição corporal com características de eficiência produtiva de fêmeas da raça Canchim
}

\author{
Fernando Baldi ${ }^{1}$, Mauricio Mello de Alencar ${ }^{2}$, Alfredo Ribeiro de Freitas ${ }^{2}$ \\ ${ }^{1}$ Programa de Pós-graduação em Zootecnia - UNESP, Campus de Jaboticabal, SP, CEP: 14884-900. \\ ${ }^{2}$ Embrapa Pecuária Sudeste, CEP: 13560-970, São Paulo, Brasil. Bolsista do CNPq.
}

RESUMO - Os objetivos neste trabalho foram estimar a herdabilidade do número (NBZ10 e NBZT) e quilogramas de bezerros desmamados (QBD10 e QBDT) pela vaca até dez anos de idade e durante a permanência da fêmea no rebanho e de quilogramas de bezerros desmamados pela vaca por ano de permanência no rebanho (QTPR) e as correlações genéticas dessas características com os pesos ajustados e não-ajustados para condição corporal, índice de tamanho corporal e condições corporais à primeira monta, ao primeiro parto e à idade adulta, idades ao primeiro, segundo e terceiro partos e tempo de permanência no rebanho. Os componentes de variância e de co-variância foram estimados pelo método da máxima verossimilhança restrita livre de derivadas, usando-se análises uni e bicaracterísticas. Os modelos estatísticos incluíram o efeito genético aditivo direto, como aleatório, e o efeito fixo de grupo de contemporâneos. As estimativas de herdabilidade obtidas das análises unicaracterísticas foram 0,10 \pm 0,05 (NBZT), 0,10 \pm 0,03 (QBDT), $0,12 \pm 0,05$ (NBZ10), 0,13 \pm 0,05 (QBD10) e 0,15 \pm 0,05 (QTPR), indicando que baixa resposta à seleção será obtida para essas características. A seleção para redução na idade ao primeiro parto não incrementará a produtividade das vacas. A seleção para aumentar o peso adulto das fêmeas acarretará redução em QTPR, NBZ10 e NBZT. A seleção para os índices de tamanho corporal ou para os pesos ajustados para condição corporal deve apresentar respostas correlacionadas com as características produtivas das fêmeas com tendências semelhantes, mas de maior magnitude que a seleção para peso da fêmea sem ajuste.

Palavras-chave: bovinos de corte, número de bezerros desmamados, parâmetros genéticos, quilogramas de bezerros desmamados, tamanho da fêmea

\section{Genetic correlations among cow body size, condition score and productive traits in Canchim cows}

\begin{abstract}
The objectives of this study were to obtain heritability estimates for number (NBZ10 and NBZT) and kilograms (QBD10 and QBDT) of calves weaned up to ten years of age and during herd life, kilograms of calves weaned per year in herd (QTPR) and their genetic correlations with culling age, ages at first, at second and at third calving, adjusted and unadjusted weights for condition score, body size indexes and condition score at first mating, at first calving and at mature age. Variance and covariance components were estimated by REML using univariate and bivariate models including the fixed effect of contemporary group and additive direct genetic and residual as random effects. Heritability estimates obtained from univariate analyses were $0.10 \pm 0.05$ (NBZT), $0.10 \pm 0.03$ (QBDT), $0.12 \pm 0.05$ (NBZ10), $0.13 \pm 0.05$ (QBD10) and $0.15 \pm 0.05$ (QTPR) and indicate low response by selection on these traits. Genetic correlation estimate suggest that selection for reducing age at first calving will not improve cow productivity. Selection for increasing mature weight will reduce kilograms of calves weaned per year in herd and the number of calves weaned up to ten years of age and during herd life. Selection on body size indexes and on cow weights adjusted for condition score may result in similar correlated responses in productive traits of cows, which would be higher than those from selection on unadjusted cow weights.
\end{abstract}

Key Words: beef cattle, cow body size, genetic parameters, number of weaned calves, kilograms of weaned calves

\section{Introdução}

A produtividade em bovinos de corte, na fase de cria, está diretamente ligada ao número e ao peso dos bezerros desmamados pela vaca. O número de bezerros está relacionado à eficiência reprodutiva da vaca, sendo dependente da idade ao primeiro parto, do intervalo de partos e do tempo de permanência no rebanho. $\mathrm{O}$ peso à desmama 
é uma característica influenciada pelo genótipo do bezerro e da mãe (Espasandin et al., 2001). O genótipo da mãe influencia por meio da produção de leite e dos cuidados maternos, além dos efeitos aditivos diretos transmitidos ao bezerro (Alencar, 1989; Albuquerque et al., 1993). Segundo Ferrel (1982), o número de bezerros desmamados é o componente que tem mais influência na produtividade do rebanho, avaliada como quilogramas de bezerro desmamado por vaca.

Apesar de sua importância, as características número e quilogramas de bezerros desmamados não são utilizadas nos programas de seleção, enquanto o peso corporal ou a taxa de crescimento a idades jovens ainda é o critério de seleção mais utilizado pela maioria dos criadores de bovinos de corte no Brasil. A seleção para maiores pesos e taxas de crescimento em idades jovens pode resultar em aumento no tamanho adulto dos animais (Silva et al., 2000; Talhari et al., 2003; Castro-Pereira et al., 2007).

Segundo Ribeiro et al. (2001), em geral, as vacas maiores dentro de uma raça ou entre raças ou cruzamentos produzem bezerros mais pesados ao desmame, porém têm maiores requerimentos de manutenção e, normalmente, produzem mais leite, o que aumenta seus requerimentos nutricionais (Kress et al., 1969; Euclides Filho et al., 1984). Porém, quando as fontes de alimentação são limitadas, as reservas corporais podem ser utilizadas para cobrir requerimentos nutricionais de manutenção e lactação, o que, dependendo do grau da restrição alimentar, pode afetar a eficiência reprodutiva e o número de bezerros produzidos (Jenkins \& Ferrel, 1992). Stewart \& Martin (1983) e Marshall et al. (1984) concluíram que existe um peso ou tamanho ótimo das vacas quanto à eficiência produtiva. A decisão sobre qual deve ser a variação aceitável do tamanho das vacas depende dos recursos genéticos e ambientais disponíveis, bem como dos custos de manutenção dos sistemas de produção de bovinos de corte.

O estudo das relações genéticas entre as características de crescimento e as medidas da eficiência produtiva das vacas é importante sob o ponto de vista do custo de manutenção do rebanho de fêmeas em crescimento e de vacas em produção. Assim, neste trabalho, objetivou-se estimar a herdabilidade das características número e quilogramas de bezerros desmamados pela vaca e as correlações genéticas dessas características com os pesos não ajustado e ajustado para condição corporal, os índices de tamanho corporal e as condições corporais à primeira monta, ao primeiro parto e à idade adulta, as idades ao primeiro, segundo e terceiro partos e o tempo de permanência no rebanho, em bovinos da raça Canchim.

\section{Material e Métodos}

Os dados utilizados neste trabalho são provenientes do rebanho da raça Canchim da Embrapa Pecuária Sudeste, localizada no município de São Carlos, São Paulo. Os animais deste rebanho foram criados em regime exclusivo de pastagens, recebendo suplementação mineral durante todo o ano.

O manejo reprodutivo do rebanho estudado variou durante o período de coleta dos dados utilizados neste trabalho. Até o ano de 1975, as novilhas entravam em reprodução com aproximadamente 34 meses de idade e $360 \mathrm{~kg}$ de peso vivo e, a partir de 1976, esses critérios foram mudados para 24 a 28 meses e aproximadamente $300 \mathrm{~kg}$ de peso vivo. Em geral, as vacas eram colocadas com touros após a desmama dos bezerros, ou seja, 7 a 8 meses após a parição. Esse tipo de manejo foi modificado em 1969, quando todas as vacas que pariram antes do início da estação de monta foram colocadas em reprodução. Nova mudança ocorreu a partir de 1976, quando todas as vacas foram colocadas com touros durante a estação de monta, com exceção daquelas prenhes da estação anterior e que iriam parir após o término da estação vigente. Em vários anos foram utilizadas duas estações de monta, uma no primeiro semestre e outra no segundo, mas que não tinham mês fixo para iniciar nem para terminar, com duração variável (2 a 4 meses).

A eliminação de vacas do rebanho se deu principalmente por motivos de doença e/ou acidente; contudo, a partir de 1977, iniciou-se o descarte de vacas consideradas de fertilidade mais baixa, ou seja, que saíssem vazias de duas estações de monta consecutivas. Procurou-se manter no rebanho apenas novilhas prenhes da primeira estação de monta.

Neste trabalho, foram estudadas as características número (NBZ10) e quilogramas (QBD10) de bezerros efetivamente desmamados pelas vacas em até 10 anos de permanência no rebanho; número (NBZT) e quilogramas (QBDT) de bezerros efetivamente desmamados durante a permanência no rebanho; e quilogramas de bezerros desmamados por ano de permanência no rebanho (QTPR). Primeiramente, foram realizadas análises de variância dos pesos à desmama dos bezerros, padronizados para 240 dias de idade, por meio do método dos quadrados mínimos e com modelo estatístico que incluiu os efeitos de ano e mês de nascimento do bezerro, sexo do bezerro e idade da vaca ao parto (efeitos linear e quadrático). Com base nessas análises, os pesos à desmama foram ajustados para ano e mês de nascimento do bezerro, sexo do bezerro e idade da vaca ao parto, antes de se calcular QBD10 e QBDT de cada fêmea, que são a soma 
dos pesos à desmama ajustados de todos os bezerros da vaca, nascidos até que ela tenha completado dez anos de idade e durante todo o tempo que ela permaneceu no rebanho, respectivamente. A característica QTPR foi obtida dividindo-se QBDT pelo tempo de permanência da vaca no rebanho (em anos).

Para as características NBZT, QBDT e QTPR, foram utilizados os dados de vacas nascidas de 1953 a 1993, uma vez que todos os animais nascidos até esse ano tinham deixado o rebanho. Para NBZ10 e QBD10, foram utilizados os dados de vacas nascidas de 1953 a 1994, porque os animais nascidos até esse ano já tinham completado dez anos de idade. Para NBZ10, QBD10 NBZT, QBDT e QTPR, foram consideradas apenas as observações de fêmeas que tiveram oportunidade de conceber e cuja saída do rebanho não foi por motivos de acidentes e, ou, doença. Foram considerados animais cujas saídas do rebanho foram por venda para abate ou reprodução e por transferência.

Foram estimadas as herdabilidades de NBZ10, QBD10, NBZT, QBDT e QTPR e as correlações genéticas dessas características com as idades ao primeiro (IDPP), segundo (IDSP) e terceiro (IDTP) partos, o tempo de permanência no rebanho (TPR), o peso (PEM), o peso ajustado para a condição corporal (PEMA), a condição corporal (CEM) e o índice de tamanho corporal (ITCEM) à entrada da primeira estação de monta, o peso (PPP), o peso ajustado para a condição corporal (PPPA), a condição corporal (CPP) e o índice de tamanho corporal (ITCPP) ao primeiro parto e o peso (PAD), o peso ajustado para a condição corporal (PADA), a condição corporal (CAD) e o índice de tamanho corporal (ITCAD) à idade adulta. Na Tabela 1 apresenta-se o número de observações e algumas estatísticas descritivas das características estudadas.

A condição corporal das vacas foi determinada usando-se uma escala de pontos que variou de 3 (muito magra) a 8 (excessivamente gorda). Os índices de tamanho corporal foram calculados padronizando-se o peso da vaca dentro de cada classe de condição corporal, conforme descrito por Baldi (2006).

Para obtenção dos parâmetros genéticos para cada característica, os componentes de variância e co-variância foram estimados pelo método de máxima verossimilhança restrita livre de derivadas, utilizando-se o programa computacional MTDFREML (Boldman et al., 1993). O modelo estatístico adotado, representado na forma matricial, foi:

$$
\mathrm{y}=\mathrm{Xb}+\mathrm{Zg}+\mathrm{e}
$$

em que $\mathrm{y}=$ vetor das variáveis dependentes; $\mathrm{b}=$ vetor de efeitos fixos de grupo de contemporâneos; $\mathrm{g}=$ vetor de efeitos genéticos aditivos diretos; e = vetor de erros aleatórios residuais associados às observações; e $\mathrm{X}$ e $\mathrm{Z}=$
Tabela 1 - Número de observações, média, desvio-padrão (DP), coeficiente de variação (CV) e valores mínimo (Min) e máximo (Max)

\begin{tabular}{lrrrrrr} 
Característica $^{1}$ & N $^{\circ}$ obs. & Média & DP & CV (\%) & Min & Max \\
\hline NBZ10 & 1.667 & 2,5 & 1,8 & 72 & 0 & 7 \\
QBD10 & 1.667 & 493 & 365 & 74 & 0 & 1.577 \\
NBZT & 1.602 & 2,8 & 2,3 & 82 & 0 & 9 \\
QBDT & 1.602 & 565 & 472 & 84 & 0 & 1.954 \\
QTPR & 1.602 & 66,1 & 39,7 & 60 & 0 & 175 \\
PEM & 1.221 & 345 & 40 & 12 & 239 & 456 \\
PEMA & 914 & 347 & 33 & 9,5 & 260 & 441 \\
CEM & 898 & 5,2 & 0,6 & 11 & 4 & 6 \\
ITCEM & 895 & 3,46 & 0,9 & 26 & 1,0 & 5,9 \\
P P & 1.095 & 426 & 51 & 12 & 300 & 565 \\
PPPA & 736 & 437 & 45 & 11 & 317 & 562 \\
CPP & 715 & 4,9 & 0,7 & 13 & 4 & 6 \\
ITCPP & 702 & 3,46 & 0,9 & 27 & 1,0 & 5,9 \\
PAD & 1.158 & 500 & 65 & 13 & 337 & 670 \\
PADA & 780 & 510 & 51 & 10 & 376 & 650 \\
CAD & 789 & 5,3 & 0,7 & 13 & 4 & 6 \\
ITCAD & 776 & 3,46 & 0,9 & 27 & 1,1 & 6,0 \\
IDPP & 1.246 & 1.144 & 105 & 9,2 & 697 & 1.400 \\
IDSP & 1.208 & 1.777 & 203 & 11 & 1.177 & 2.190 \\
IDTP & 1.031 & 2.345 & 333 & 14 & 1.587 & 3.292 \\
TPR & 1.630 & 2.617 & 1.278 & 49 & 746 & 5.973 \\
\hline
\end{tabular}

${ }^{1}$ Número (NBZ10, NBZT) e quilogramas (QBD10, QBDT) de bezerros efetivamente desmamados pelas vacas em até 10 anos de permanência no rebanho e durante a permanência no rebanho e quilogramas de bezerros desmamados por ano de permanência no rebanho (QTPR), idades ao primeiro (IDPP), segundo (IDSP) e terceiro (IDTP) partos, tempo de permanência no rebanho (TPR), peso (PEM), peso ajustado para a condição corporal (PEMA), condição corporal (CEM) e índice de tamanho corporal (ITCEM) à entrada da primeira estação de monta, peso (PPP), peso ajustado para a condição corporal (PPPA), condição corporal (CPP) e índice de tamanho corporal (ITCPP) ao primeiro parto, peso (PAD), peso ajustado para a condição corporal (PADA), condição corporal (CAD) e índice de tamanho corporal (ITCAD) à idade adulta.

matrizes de incidência para os efeitos fixos e aleatórios aditivos diretos, respectivamente.

As pressuposições acerca da distribuição de $\mathbf{y}$, g e e podem ser descritas como:

$$
\left[\begin{array}{l}
y \\
g \\
e
\end{array}\right] \sim \mathbb{N}\left\{\left[\begin{array}{c}
\boldsymbol{X} \beta \\
\boldsymbol{0} \\
\boldsymbol{0}
\end{array}\right],\left[\begin{array}{ccc}
Z \mathbf{G} \mathbf{Z}^{\prime}+\boldsymbol{R} & \boldsymbol{Z G} & \boldsymbol{R} \\
\mathbf{G} \mathbf{Z}^{\prime} & \boldsymbol{G} & \Phi \\
\boldsymbol{R} & \Phi & \boldsymbol{R}
\end{array}\right]\right\}
$$

em que $\boldsymbol{G}$ é a matriz de variâncias e co-variâncias dos efeitos aleatórios do vetor $\boldsymbol{g}$ e $\boldsymbol{R}$, a matriz de variâncias e co-variâncias residuais.

Este modelo foi aplicado para todos os caracteres estudados, havendo variação apenas dos efeitos fixos, conforme a característica sob análise. Para estimação dos componentes de variância e da herdabilidade de cada variável, foram realizadas análises unicaracterísticas e para estimação das correlações genéticas entre as características, análises bicaracterísticas. As médias das variâncias genéticas e fenotípicas para cada característica, obtidas 
nas análises bicaracterísticas, foram utilizadas para estimação da herdabilidade, que, posteriormente, foi comparada com a estimativa de herdabilidade obtida pela análise unicaracterística. Foi considerado em todas as análises o critério de convergência de $10^{-9}$.

O grupo de contemporâneos (GC) para NBZT, QBDT, NBZ10, QBD10 e QTPR foi definido apenas como ano de nascimento da fêmea. Os GC para as características PEM, PEMA, CEM, ITCEM, PPP, PPPA, CPP, ITCPP, PAD, PADA, CAD e ITCAD foram definidos pelo ano e mês de início da primeira estação de monta, para as quatro primeiras, e ano e mês de parto, para as oito últimas. Para IDPP os GC foram elaborados pelo ano e mês de nascimento; para IDSP, apenas pelo ano do primeiro parto; para IDTP, pelo ano e mês do segundo parto; e para TPR, pelo ano e mês de nascimento da vaca. Foram considerados GC com no mínimo duas observações. Em todas as análises, foi utilizado um arquivo de "pedigree" contendo identificação do animal, pai e mãe, totalizando 12.311 animais na matriz de parentesco.

\section{Resultados e Discussão}

Com base nas estimativas dos componentes de variância genética aditiva, residual e fenotípica e dos coeficientes de herdabilidade das características estudadas, obtidas pelas análises unicaracterísticas e bicaracterísticas (Tabela 2), nota-se que, para todas as características, as estimativas de herdabilidade obtidas pelas análises unicaracterísticas foram semelhantes às obtidas pelas análises bicaracterísticas.

As estimativas de herdabilidade para todas as características apresentaram valores, em geral, baixos, variando de 0,10 a 0,15 (Tabela 2). As variâncias genética aditiva, residual e fenotípica foram inferiores para NBZ10 e QBD10 em relação às obtidas para NBZT e QBDT, no entanto, não houve diferenças entre as estimativas de herdabilidade. Meyer (1990) $(0,07$ e 0,09) e Mercadante et al. (1996)
(0,07), pelo método REML, obtiveram estimativas de herdabilidade para NBZT próximas à obtida neste estudo. Contudo, Tanida et al. (1988), para NBZT $(0,22)$ nas raças Angus e Hereford, utilizando o método dos quadrados mínimos, Gianlorenço et al. (2003), para NBZ10 $(0,36)$ e QBD10 (0,41), e Mello et al. (2006), para NBZT $(0,23)$, QBDT $(0,32)$, NBZ10 $(0,22)$ e QBD10 $(0,24)$, usando inferência bayesiana, relataram estimativas superiores.

Características indicadoras de produtividade nas fêmeas, como as adotadas neste estudo, têm estreita ligação com as características reprodutivas, caracterizadas por herdabilidade de magnitude baixa. Os coeficientes de herdabilidade obtidos neste trabalho sugerem que há pouco campo para seleção para essas características, além do fato de serem características medidas tardiamente na vida do animal.

Analisando as estimativas dos componentes de covariâncias e das correlações genéticas entre as características produtivas das fêmeas com o tempo de permanência no rebanho (TPR) e as idades ao primeiro (IDPP), segundo (IDSP) e terceiro (IDTP) partos (Tabela 2), observa-se que as correlações entre as características produtivas e as idades ao parto aumentaram com a ordem do parto. As correlações entre IDPP e as características produtivas foram baixas, enquanto as correlações entre IDSP e IDTP e as características produtivas foram de moderadas a altas. Este resultado é esperado, uma vez que a proximidade entre as características é maior, ou seja, uma vaca que atinge o terceiro parto é provável que tenha maior produtividade em relação a uma vaca de primeiro parto. Mello et al. (2006), utilizando o mesmo banco de dados e inferência bayesiana, relataram correlações superiores entre IDPP e NBZT $(-0,43)$, QBDT(-0,46) e QBD10 $(-0,47)$ e semelhantes com NBZ10 $(-0,33)$, em relação às obtidas neste trabalho, na raça Canchim. Mercadante et al. (1996), na raça Nelore, usando REML, estimaram correlação de -0,44 do número de bezerros com a idade ao primeiro parto.

Tabela 2 - Estimativas dos componentes de variância genética aditiva $\left(\hat{\sigma}_{a}^{2}\right)$, residual $\left(\hat{\sigma}_{e}^{2}\right)$ e fenotípica $\left(\hat{\sigma}_{p}^{2}\right)$ e dos coeficientes de herdabilidade $\left(\hat{h}^{2}\right)$ obtidas em análises unicaracterísticas e valores médio $\left(\hat{h}_{b}^{2}\right)$, máximo $\left(\max \hat{h}_{b}{ }^{2}\right)$ e mínimo $\left(\min \hat{h}_{b}{ }^{2}\right)$ dos coeficientes de herdabilidade obtidos em análises bicaracterísticas, para as características estudadas

\begin{tabular}{|c|c|c|c|c|c|c|c|}
\hline Característica $^{1}$ & $\hat{\sigma}_{a}^{2}$ & $\hat{\sigma}_{e}^{2}$ & $\hat{\sigma}_{p}^{2}$ & $\hat{h}^{2}$ & $\hat{h}_{b}^{2}$ & $\max \hat{h}_{b}^{2}$ & $\min \hat{h}_{b}{ }^{2}$ \\
\hline NBZT & 0,5 & 4,7 & 5,2 & $0,10 \pm 0,05$ & 0,11 & 0,13 & 0,10 \\
\hline QBDT & 21.473 & 192.504 & 213.977 & $0,10 \pm 0,03$ & 0,09 & 0,12 & 0,09 \\
\hline NBZ10 & 0,4 & 2,6 & 3,0 & $0,12 \pm 0,05$ & 0,12 & 0,13 & 0,11 \\
\hline QBD10 & 15.329 & 106.886 & 122.215 & $0,13 \pm 0,05$ & 0,12 & 0,13 & 0,12 \\
\hline QTPR & 220 & 1.233 & 1.452 & $0,15 \pm 0,05$ & 0,15 & 0,17 & 0,14 \\
\hline
\end{tabular}

${ }^{1}$ NBZT e QBDT: número e quilogramas de bezerros efetivamente desmamados durante a permanência da vaca no rebanho; NBZ10 e QBD10: número e quilogramas de bezerros efetivamente desmamados em até 10 anos de permanência da vaca no rebanho; QTPR: quilogramas de bezerros desmamados por ano de permanência da vaca no rebanho. 
As estimativas de correlação genética obtidas neste trabalho sugerem que a seleção para menor IDPP não deve alterar o número e quilogramas de bezerros desmamados até os dez anos de idade ou durante toda a permanência da vaca no rebanho, mas a seleção para reduzir as idades ao segundo e ao terceiro parto deve resultar em respostas correlacionadas favoráveis nas características de produtividade.

As correlações entre as características produtivas e TPR foram positivas e altas (Tabela 3), indicando que o aumento na longevidade das vacas resultará em incremento na produtividade. Este resultado é lógico, uma vez que vacas que permanecem por mais tempo no rebanho têm maior oportunidade de desmamar maior número e quilogramas de bezerros. O inverso também é verdadeiro, ou seja, as vacas permanecem mais tempo no rebanho, pois são mais produtivas. Mercadante et al. (1996), em bovinos Nelore, estimaram correlação genética de 0,98 do número de bezerros com o tempo de permanência no rebanho.

As magnitudes das correlações entre os pesos e as características produtivas foram maiores à idade adulta (Tabela 4). As correlações de PEM e PPP com as características produtivas foram baixas a nulas. Para PAD, as correlações foram baixas com QBDT e QBD10 e moderadas com NBZT, NBZ10 e QTPR (Tabela 4). Mello et al. (2006), utilizando fêmeas da raça Canchim e inferência bayesiana, relataram valores de correlações entre o peso adulto das fêmeas e as características produtivas inferiores aos obtidos neste estudo. Gianlorenço et al. (2003) estimaram as correlações do peso de machos aos 12 meses de idade com o número e quilogramas de bezerros desmamados pelas fêmeas em até dez anos de idade, em um rebanho da raça Canchim, obtendo valores de 0,38 e 0,61, respectivamente. Tanida et al. (1988) também observaram correlação genética favorável $(0,72)$ entre o número de bezerros desmamados e o peso ao desmame de fêmeas da raça Hereford.

Observa-se, portanto, que, de acordo com a literatura, a idades jovens, a seleção para maior peso corporal, tanto em machos como em fêmeas, possui associação genética favorável com as características produtivas das fêmeas. Contudo, de acordo com os resultados obtidos neste trabalho, em idades mais avançadas, as correlações genéticas dos pesos com as características produtivas são nulas ou desfavoráveis. A seleção para maiores pesos das fêmeas à primeira monta e ao primeiro parto parece não comprometer o desempenho quanto às características produtivas, mas o aumento do peso adulto deve influenciar desfavoravelmente as características NBZT, NBZ10 e QTPR.

As correlações entre a condição corporal e as características produtivas foram variáveis (Tabela 4). As correlações genéticas entre CAD e as características produtivas foram de baixas a nulas. No entanto, as correlações de CEM com NBZT, QBDT e QTPR foram moderadas, mas baixas com NBZ10 e QBD10. Já as correlações de CPP com as características produtivas foram de moderadas a altas. Mello et al. (2004) relataram correlações genéticas praticamente nulas entre CPP e características produtivas.

De acordo com Wiltbank et al. (1962) e Bellows \& Short (1978), as vacas com maior condição corporal ao parto possuem anestro pós-parto menor. Portanto, é provável que as vacas com maior CPP apresentem menor período de anestro após o primeiro parto e, portanto, têm maior probabilidade de conceber e permanecer no rebanho produtivamente. Os resultados obtidos neste trabalho, em geral, sugerem que animais com melhor condição corporal à primeira monta e ao primeiro parto devem ser mais produtivos em termos de número e quilogramas de bezerros desmamados.

A magnitude das correlações entre os índices de tamanho (Tabela 5) e as características produtivas foi maior que aquela obtida entre os pesos (Tabela 4) e as características produtivas. Semelhantemente às correlações obtidas entre o peso adulto e as características produtivas, o aumento no tamanho adulto das fêmeas levará à queda nas medidas de eficiência produtiva das fêmeas.

Tabela 3 - Estimativas de covariâncias $\left(\sigma_{\mathrm{a} 1 \mathrm{a} 2}\right)$ e das correlações genéticas $\left(\mathrm{r}_{\mathrm{a} 1 \mathrm{a} 2}\right)$ do tempo de permanência no rebanho (TPR) e das idades ao primeiro (IDPP), segundo (IDSP) e terceiro (IDTP) partos com as características produtivas das fêmeas, em análise bicaracterística

Característica

\begin{tabular}{|c|c|c|c|c|c|c|c|c|}
\hline \multirow[b]{2}{*}{ Característica $^{1}$} & \multicolumn{2}{|c|}{ IDPP } & \multicolumn{2}{|c|}{ IDSP } & \multicolumn{2}{|c|}{ IDTP } & \multicolumn{2}{|c|}{ TPR } \\
\hline & $\sigma_{\mathrm{a} 1 \mathrm{a} 2}$ & $\mathrm{r}_{\mathrm{a} 1 \mathrm{a} 2}$ & $\sigma_{\mathrm{a} 1 \mathrm{a} 2}$ & $\mathrm{r}_{\mathrm{a} 1 \mathrm{a} 2}$ & $\sigma_{\mathrm{a} 1 \mathrm{a} 2}$ & $\mathrm{r}_{\mathrm{a} 1 \mathrm{a} 2}$ & $\sigma_{\mathrm{a} 1 \mathrm{a} 2}$ & $\mathrm{r}_{\mathrm{a} 1 \mathrm{a} 2}$ \\
\hline NBZT & -4 & $-0,26$ & -21 & $-0,65$ & -47 & $-0,66$ & 226 & 0,96 \\
\hline QBDT & -978 & $-0,30$ & -4.658 & $-0,66$ & -9.331 & $-0,66$ & 43.765 & 0,95 \\
\hline NBZ10 & -5 & $-0,33$ & -24 & $-0,84$ & -41 & $-0,71$ & 189 & 0,93 \\
\hline QBD10 & -972 & $-0,34$ & -4.994 & $-0,87$ & -8.440 & $-0,72$ & 37.019 & 0,91 \\
\hline
\end{tabular}

${ }^{1}$ ND10 e QD10: número e quilogramas de bezerros efetivamente desmamados em até 10 anos de permanência da vaca no rebanho; NBZT e QBDT: número e quilogramas de bezerros efetivamente desmamados durante a permanência da vaca no rebanho. 
Tabela 4 - Estimativas das correlações genéticas dos pesos à primeira monta (PEM), ao primeiro parto (PPP) e à idade adulta (PAD) e das condições à primeira monta (CEM), ao primeiro parto (CPP) e à idade adulta (CAD) com as características produtivas das fêmeas, em análise bicaracterística

\begin{tabular}{lrrrrrr}
\hline & \multicolumn{6}{c}{ Característica } \\
\cline { 2 - 7 } Caract. $^{1}$ & PEM & P P & PAD & CEM & CPP & CAD \\
\hline NBZT & $-0,36$ & $-0,22$ & $-0,47$ & 0,49 & 0,81 & $-0,23$ \\
QBDT & $-0,05$ & $-0,07$ & $-0,36$ & 0,42 & 0,76 & $-0,14$ \\
NBZ10 & $-0,32$ & 0,03 & $-0,43$ & 0,23 & 0,51 & 0,00 \\
QBD10 & $-0,03$ & $-0,02$ & $-0,32$ & 0,19 & 0,47 & $-0,05$ \\
QTPR & 0,05 & 0,04 & $-0,40$ & 0,43 & 0,61 & $-0,08$ \\
\hline
\end{tabular}

${ }_{1}$ NBZT e QBDT: número e quilogramas de bezerros efetivamente desmamados durante a permanência da vaca no rebanho; NBZ10 e QBD10: número e quilogramas de bezerros efetivamente desmamados em até 10 anos de permanência da vaca no rebanho; QTPR: quilogramas de bezerros desmamados por ano de permanência da vaca no rebanho.

Tabela 5 - Estimativas das correlações genéticas do índice de tamanho corporal à primeira monta (ITCPM), ao primeiro parto (ITCPP) e à idade adulta (ITCAD) e dos pesos ajustados para condição corporal à primeira monta (PEMA), ao primeiro parto (PPPA) e à idade adulta (PADA) com as características produtivas das fêmeas, em análise bicaracterística

\begin{tabular}{lcccccc}
\hline & \multicolumn{5}{c}{ Característica } \\
\cline { 2 - 7 } Caract. $^{1}$ & ITCPM & ITCPP & ITCAD & PEMA & PPPA & PADA \\
\hline NBZT & $-0,70$ & $-0,75$ & $-0,93$ & $-0,73$ & $-0,70$ & $-0,91$ \\
QBDT & $-0,39$ & $-0,53$ & $-0,83$ & $-0,39$ & $-0,50$ & $-0,82$ \\
NBZ10 & $-0,44$ & $-0,58$ & $-0,98$ & $-0,33$ & $-0,58$ & $-0,93$ \\
QBD10 & $-0,12$ & 0,16 & $-0,87$ & $-0,03$ & $-0,38$ & $-0,80$ \\
QTPR & $-0,03$ & $-0,22$ & $-0,64$ & $-0,03$ & $-0,25$ & $-0,64$
\end{tabular}

${ }^{1}$ NBZT e QBDT: número e quilogramas de bezerros efetivamente desmamados durante a permanência da vaca no rebanho; NBZ10 e QBD10: número e quilogramas de bezerros efetivamente desmamados em até 10 anos de permanência da vaca no rebanho; QTPR: quilogramas de bezerros desmamados por ano de permanência da vaca no rebanho.

Verifica-se, na Tabela 5, que as correlações genéticas dos pesos ajustados para condição corporal com as características produtivas foram semelhantes àquelas obtidas entre os índices de tamanho corporal e as características produtivas, resultados esperados, pois os pesos ajustados para a condição corporal e os índices de tamanho corporal podem ser consideradas como sendo as mesmas características (Baldi, 2006).

\section{Conclusões}

Para as condições de criação em que foram coletados os dados deste trabalho, as características produtivas estudadas dificilmente responderão à seleção. A seleção para reduzir a idade ao primeiro parto não acarretará incremento na produtividade das vacas, contudo, o aumento na longevidade das matrizes levará a incremento em sua produtividade.
A seleção para maiores pesos à primeira monta e ao primeiro parto não deve ocasionar mudanças nas características produtivas. No entanto, a seleção para maior peso adulto deve alterar desfavoravelmente o número de bezerros desmamados pela vaca até dez anos de idade ou durante sua permanência no rebanho e a característica quilograma de bezerros desmamados pela vaca por ano de permanência no rebanho.

Em termos gerais, espera-se que as melhorias nos índices de tamanho corporal ou nos pesos ajustados para condição corporal apresentem respostas correlacionadas nas características produtivas das fêmeas com tendências semelhantes, mas de maior magnitude em relação à seleção para peso da fêmea não ajustado para condição corporal.

\section{Literatura Citada}

ALBUQUERQUE, L.G.; ELER, J.P.; COSTA, M.R.P. Produção de leite e desempenho do bezerro na fase de aleitamento em três raças bovinas de corte. Revista da Sociedade Brasileira de Zootecnia, v.22, n.5, p.745-754, 1993.

ALENCAR, M.M. Relação entre produção de leite da vaca e desempenho do bezerro nas raças Canchim e Nelore. Revista da Sociedade Brasileira de Zootecnia, v.18, n.2, p.146156, 1989.

BALDI, F.S. Relação genética de características de tamanho corporal com características de eficiência reprodutiva e produtiva de fêmeas da raça Canchim. Jaboticabal: Universidade Estadual Paulista, 2006. 97p. Dissertação (Mestrado em Genética e Melhoramento Animal) - Universidade Estadual Paulista, 2006.

BELLOWS, R.A.; SHORT, R.E. Effects of pre-calving feed level on birth weight, calving difficulty and subsequent fertility. Journal of Animal Science, v.46, n.5, p.1522-1530, 1978.

BOLDMAN, K.G.; KRIESE, L.A.; van VLECK, L.D. et al. A manual for use of MTDFREML. Clay Center: USDA-ARS, 1993. 120p.

CASTRO-PEREIRA, V.M.; ALENCAR, M.M.; BARBOSA, P.F. Estimativas de parâmetros genéticos e de ganhos direto e indireto à seleção para características de crescimento de machos e fêmeas da raça Canchim. Revista Brasileira de Zootecnia, v.36, n.4, p.1037-1044, 2007 (supl.).

ESPASANDIN, A.C.; PACKER, I.U.; ALENCAR, M.M. Produção de leite e comportamento de amamentação em cinco sistemas de produção de gado de corte. Revista Brasileira Zootecnia, v.30, n.3, p.702-708, 2001.

EUCLIDES FILHO, K.; RESTLE, J.; OLSON, T.A. Medidas de eficiência na produção de terneiros a partir de vacas de tamanho e habilidade leiteira diferentes. In: REUNIÃO ANUAL DA SOCIEDADE BRASILEIRA DE ZOOTECNIA, 21., 1984, Belo Horizonte. Anais... Belo Horizonte: Sociedade Brasileira de Zootecnia, 1984. p.138.

FERRELL, C.L. Effects of postweaning rate of gain on onset of puberty and productive performance of heifers of different breeds. Journal of Animal Science, v.55, n.5, p.1272-1283, 1982.

GIANLORENCO, V.K.; ALENCAR, M.M.; TORAL, F.L.B. Herdabilidades e correlações genéticas de características de machos e fêmeas, em um rebanho bovino da raça Canchim. Revista Brasileira de Zootecnia, v.32, n.6, p.1587-1593, 2003 (supl.1).

JENKINS, T.G.; FERREL, C.L. Lactation characteristics of nine breeds of cattle fed various quantities of dietary energy. Journal of Animal Science, v.70, n.6, p.1652-1660, 1992. 
KRESS, D.D.; HAUSER, E.R.; CHAPMAN, A.B. Efficiency of production and cow size in beef cattle. Journal of Animal Science, v.29, n.2, p.373-383, 1969.

MARSHALL, T.E.; MOHLER, M.A.; STEWART, T.S. Relationship of lifetime productivity with mature weight and maturation rate in Red Poll cows. Animal Production, v.39, n.2, p.383-387, 1984.

MELLO, S.P.; ALENCAR, M.M.; TORAL, F.B. et al. Análise genética de características de crescimento e de produtividade em vacas da raça Canchim. In: REUNIÃO ANUAL DA SOCIEDADE BRASILEIRA DE ZOOTECNIA, 41., 2004, Campo Grande. Anais... Campo Grande: Sociedade Brasileira de Zootecnia, 2004. (CD-ROM).

MELLO, S.P.; ALENCAR, M.M.; TORAL, F.L.B. et al. Estimativas de parâmetros genéticos para características de crescimento e produtivas em vacas da raça Canchim, utilizando-se inferência bayesiana. Revista Brasileira de Zootecnia, v.35, n.1, p.9297, 2006.

MERCADANTE, M.E.Z.; LÔBO, R.B.; BORJAS, et al. Estudo genético de características indicadoras da vida útil de fêmeas de um rebanho da raça Nelore. In: SIMPÓSIO NACIONAL DE MELHORAMENTO ANIMAL, 1., 1996, Ribeirão Preto. Anais... Ribeirão Preto: Sociedade Brasileira de Melhoramento Animal, 1996. p.260-262.

MEYER, K.; HAMMOND, K.; PARNELL, P.F. et al. Genetic parameters for reproductive traits in Australian beef cattle. In:
AUSTRALIAN ASSOCIATION OF ANIMAL BREEDING AND GENETICS, 8., 1990, Armidale. Proceedings... Armidale: Association for the Advancement of Animal Breeding and Genetics, 1990. p.389-392.

RIBEIRO, E.L.A.; RESTLE, J.; ROCHA, M.A. Productive efficiency of Angus and Charolais primiparous cows. Revista Brasileira de Zootecnia, v.30, n.1, p.125-132, 2001

SILVA, A.M.; ALENCAR, M.M.; FREITAS, A.R. Herdabilidade e correlações genéticas para peso e perímetro escrotal de machos e características reprodutivas e de crescimento de fêmeas, na raça Canchim. Revista Brasileira de Zootecnia, v.29, p.22232230, 2000.

STEWART, T.S.; MARTIN, T.G. Optimal mature size of Angus cows for maximum cow productivity. Animal Production, v.37, p.179-182, 1983.

TANIDA, H.; HOHENBOKEN, W.D.; DENISE, S.K. Genetic aspects of longevity in Angus and Hereford cows. Journal of Animal Science, v.66, n.3, p.640-647, 1988.

TALHARI, F.M.; ALENCAR, M.M.; MASCIOLI, A.S. Correlações genéticas entre características produtivas das fêmeas em um rebanho da Raça Canchim. Revista Brasileira de Zootecnia, v.32, n.4, p.880-886, 2003.

WILTBANK, J.N.; ROWDEN, W.W.; INGALLS, J.E. et al. Effect of energy level on reproductive phenomena of mature Hereford cows. Journal of Animal Science, v.21, n.1, p.219-226, 1962 